\title{
The Ramsey number of loose triangles and quadrangles in hypergraphs
}

\author{
Andras Gyárfás* \\ Computer and Automation Research Institute \\ Hungarian Academy of Sciences \\ Budapest, P.O. Box 63, Hungary, H-1518 \\ gyarfas@sztaki.hu
}

\author{
Ghaffar Raeisi \\ Department of Mathematical Sciences \\ Isfahan University of Technology \\ Isfahan, 84156-83111, Iran \\ g.raeisi@math.iut.ac.ir
}

Submitted: Apr 5, 2011; Accepted: May 11, 2012; Published: Jun 6, 2012

Mathematics Subject Classifications: 05C15, 05C55, 05C65

\begin{abstract}
Asymptotic values of hypergraph Ramsey numbers for loose cycles (and paths) were determined recently. Here we determine some of them exactly, for example the 2-color hypergraph Ramsey number of a $k$-uniform loose 3 -cycle or 4-cycle: $R\left(\mathcal{C}_{3}^{k}, \mathcal{C}_{3}^{k}\right)=3 k-2$ and $R\left(\mathcal{C}_{4}^{k}, \mathcal{C}_{4}^{k}\right)=4 k-3$ (for $k \geqslant 3$ ). For more than 3 colors we could prove only that $R\left(\mathcal{C}_{3}^{3}, \mathcal{C}_{3}^{3}, \mathcal{C}_{3}^{3}\right)=8$. Nevertheless, the $r$-color Ramsey number of triangles for hypergraphs are much smaller than for graphs: for $r \geqslant 3$,

$$
r+5 \leqslant R\left(\mathcal{C}_{3}^{3}, \mathcal{C}_{3}^{3}, \ldots, \mathcal{C}_{3}^{3}\right) \leqslant 3 r
$$
\end{abstract}

Keywords: Hypergraph Ramsey Number, Loose Cycle, Loose Path.

\section{$1 \quad$ Introduction}

In this paper, we consider the problem of finding the 2-color Ramsey number for uniform hypergraph paths and cycles. There are several natural definitions for a cycle and a path in a uniform hypergraph. The one we focus on here is called loose. The $k$-uniform loose cycle $\mathcal{C}_{n}^{k}$ (shortly, a cycle of length $n$ ), is the hypergraph with vertex set $\left\{v_{1}, v_{2}, \ldots, v_{n(k-1)}\right\}$ and with the set of $n$ hyperedges $e_{i}=\left\{v_{1}, v_{2}, \ldots, v_{k}\right\}+i(k-1)$, $i=0,1, \ldots, n-1$, where we use $\bmod n$ arithmetic and adding a number $t$ to a set $H=\left\{v_{1}, v_{2}, \ldots, v_{k}\right\}$ means a shift, i.e. the set obtained by adding $t$ to subscripts of each element of $H$. Similarly, the $k$-uniform loose path $\mathcal{P}_{n}^{k}$ (simply, a path of length $n$ ), is the hypergraph with vertex set $\left\{v_{1}, v_{2}, \ldots, v_{n(k-1)+1}\right\}$ and with the set of $n$ hyperedges

*Supported in part by OTKA grant K68322. 
$e_{i}=\left\{v_{1}, v_{2}, \ldots, v_{k}\right\}+i(k-1), i=0,1, \ldots, n-1$. Also by a star $\mathcal{S}_{n}^{k}$ we mean the $k$ uniform hypergraph with vertex set $\left\{v, v_{1}, v_{2}, \ldots, v_{n(k-1)}\right\}$ and with the set of $n$ edges $e_{i}=\{v\} \cup\left\{v_{1}, v_{2}, \ldots, v_{k-1}\right\}+i(k-1), i=0,1, \ldots, n-1$. For $k=2$ we get the definition of the cycle $C_{n}$, the path $P_{n}$ and the star $K_{1, n}$ with $n$ edges. A classical result in graph Ramsey theory (see [5]) states that $R\left(P_{n}, P_{m}\right)=n+\left\lfloor\frac{m+1}{2}\right\rfloor$, where $n \geqslant m \geqslant 1$. Also it is well-known (see [3, 4]) that for every $n \geqslant m$ and even $m, R\left(P_{n}, C_{m}\right)=R\left(P_{n}, P_{m}\right)=n+\frac{m}{2}$.

For given $k$-uniform hypergraphs $\mathcal{H}_{1}, \mathcal{H}_{2}, \ldots, \mathcal{H}_{t}$ the Ramsey number $R\left(\mathcal{H}_{1}, \mathcal{H}_{2}, \ldots, \mathcal{H}_{t}\right)$ is the smallest integer $N$ such that in every $t$-coloring of the edges of the complete $k$-uniform hypergraph $\mathcal{K}_{N}^{k}$ there is a monochromatic copy of $\mathcal{H}_{i}$ in color $i$, for some $i, 1 \leqslant i \leqslant t$. It was proved [8] that $R\left(\mathcal{C}_{n}^{3}, \mathcal{C}_{n}^{3}\right)$ is asymptotically equal to $\frac{5 n}{2}$. Subsequently, Gyárfás et. al. in [6] extended this result to the $k$-uniform loose cycles and proved that $R\left(\mathcal{C}_{n}^{k}, \mathcal{C}_{n}^{k}\right)$ is asymptotically equal to $\frac{1}{2}(2 k-1) n$. Here we determine the exact value of the 2-color Ramsey number of a $k$-uniform loose triangle and quadrangle, $R\left(\mathcal{C}_{3}^{k}, \mathcal{C}_{3}^{k}\right)=3 k-2$ (Theorem 5) and $R\left(\mathcal{C}_{4}^{k}, \mathcal{C}_{4}^{k}\right)=4 k-3$ (Theorem 9). We observe that the $r$-color Ramsey number of loose triangles in 3-uniform hypergraphs is linear, in contrast to the graph case, when it is at least exponential (Proposition 13).

Perhaps the following lower bound always gives the exact value of the 2-color hypergraph Ramsey numbers for loose paths and cycles. This is certainly the case for all results of this paper, therefore we shall always prove just the claimed upper bounds for the Ramsey numbers. During this paper, for a 2-edge coloring of a uniform hypergraph $\mathcal{H}$, say red and blue, we denote by $\mathcal{F}_{\text {red }}$ and $\mathcal{F}_{\text {blue }}$ the induced hypergraph on edges of color red and blue, respectively.

Lemma 1. For every $n \geqslant m \geqslant 2$ and $k \geqslant 3,(k-1) n+\left\lfloor\frac{m+1}{2}\right\rfloor$ is a lower bound for both $R\left(\mathcal{P}_{n}^{k}, \mathcal{P}_{m}^{k}\right)$ and $R\left(\mathcal{P}_{n}^{k}, \mathcal{C}_{m}^{k}\right)$. Moreover, $R\left(\mathcal{C}_{n}^{k}, \mathcal{C}_{m}^{k}\right) \geqslant(k-1) n+\left\lfloor\frac{m+1}{2}\right\rfloor-1$.

Proof. To prove that $(k-1) n+\left\lfloor\frac{m+1}{2}\right\rfloor$ is a lower bound for both Ramsey numbers $R\left(\mathcal{P}_{n}^{k}, \mathcal{P}_{m}^{k}\right)$ and $R\left(\mathcal{P}_{n}^{k}, \mathcal{C}_{m}^{k}\right)$, we exhibit a 2-coloring of the edges of the complete $k$-uniform hypergraph on $(k-1) n+\left\lfloor\frac{m+1}{2}\right\rfloor-1$ vertices such that $\mathcal{P}_{n}^{k} \nsubseteq \mathcal{F}_{\text {red }}$ and $\mathcal{P}_{m}^{k} \nsubseteq \mathcal{F}_{\text {blue }}$ (also $\left.\mathcal{C}_{m}^{k} \nsubseteq \mathcal{F}_{\text {blue }}\right)$. For this purpose, Partition the vertex set into parts $A$ and $B$, where $|A|=(k-1) n$ and $|B|=\left\lfloor\frac{m+1}{2}\right\rfloor-1$. We color all edges that contain a vertex of $B$ blue, and the rest red. Now, this coloring can not contain a red copy of $\mathcal{P}_{n}^{k}$, since any such copy must have all vertices in $A$ and $|A|=(k-1) n$. Clearly a longest blue path (also a longest blue cycle) has length at most $m-1$, as claimed. By the same argument and considering parts $A$ and $B$ with $|A|=(k-1) n-1$ and $|B|=\left\lfloor\frac{m+1}{2}\right\rfloor-1$, we obtain that $(k-1) n+\left\lfloor\frac{m+1}{2}\right\rfloor-1 \leqslant R\left(\mathcal{C}_{n}^{k}, \mathcal{C}_{m}^{k}\right)$, which completes the proof.

\section{Ramsey number of short paths and cycles}

The following lemma shows an easy but useful property of monochromatic cycles in uniform hypergraphs on $R\left(\mathcal{P}_{n}^{k}, \mathcal{P}_{m}^{k}\right)$ vertices. 
Lemma 2. Let $n \geqslant m \geqslant 3$ and $\mathcal{K}_{(k-1) n+\left\lfloor\frac{m+1}{2}\right\rfloor}^{k}$ be 2-edge colored red and blue. If $\mathcal{C}_{n}^{k} \subseteq \mathcal{F}_{\text {red }}$ then either $\mathcal{P}_{n}^{k} \subseteq \mathcal{F}_{\text {red }}$ or $\mathcal{P}_{m}^{k} \subseteq \mathcal{F}_{\text {blue }}$. Also, if $\mathcal{C}_{n}^{k} \subseteq \mathcal{F}_{\text {red }}$ then either $\mathcal{P}_{n}^{k} \subseteq \mathcal{F}_{\text {red }}$ or $\mathcal{C}_{m}^{k} \subseteq \mathcal{F}_{\text {blue }}$.

Proof. Let $e_{i}=\left\{v_{1}, v_{2}, \ldots, v_{k}\right\}+i(k-1)(\bmod n), i=0,1, \ldots, n-1$, be the edges of $\mathcal{C}_{n}^{k} \subseteq \mathcal{F}_{\text {red }}$ and $W=\left\{x_{1}, x_{2}, \ldots, x_{\left\lfloor\frac{m+1}{2}\right\rfloor}\right\}$ be the set of the remaining vertices. Let $1 \leqslant i \leqslant m-1$ and set $e_{0}^{\prime}=\left(e_{0} \backslash\left\{v_{1}\right\}\right) \cup\left\{x_{1}\right\}, e_{i}^{\prime}=\left(e_{i} \backslash\left\{v_{(i+1)(k-1)+1}\right\}\right) \cup\left\{x_{\frac{i+2}{2}}\right\}$ if $i$ is even and $e_{i}^{\prime}=\left(e_{i} \backslash\left\{v_{i(k-1)+1}\right\}\right) \cup\left\{x_{\frac{i+1}{2}}\right\}$ if $i$ is odd. If one of $e_{i}^{\prime}$ is red, we have a monochromatic $\mathcal{P}_{n}^{k} \subseteq \mathcal{F}_{\text {red }}$, otherwise $e_{0}^{\prime} e_{1}^{\prime} \ldots e_{m-1}^{\prime}$ form a $\mathcal{P}_{m}^{k} \subseteq \mathcal{F}_{\text {blue }}$, which completes the proof of the first part. For the second part, for even $m$ replace the hyperedges $e_{0}^{\prime}$ and $e_{m-1}^{\prime}$ by $\left(e_{0} \backslash\left\{v_{k}\right\}\right) \cup\left\{x_{1}\right\}$ and $\left(e_{n-1} \backslash\left\{v_{(n-1)(k-1)+1}\right\}\right) \cup\left\{x_{\frac{m}{2}}\right\}$, respectively, and for odd $m$ replace $e_{0}^{\prime}$ by $\left(e_{0} \backslash\left\{v_{k-1}, v_{k}\right\}\right) \cup\left\{x_{1}, x_{\frac{m+1}{2}}\right\}$. By a similar argument, $e_{0}^{\prime} e_{1}^{\prime} \ldots e_{m-1}^{\prime}$ form a $\mathcal{C}_{m}^{k} \subseteq \mathcal{F}_{\text {blue }}$, which completes the proof.

Another tool is a simple but useful folkloristic remark.

Remark 3. If a $k$-uniform complete hypergraph is 2-colored and both colors are used at least once, then there are two edges of distinct colors intersecting in $k-1$ vertices.

Proof. Select a red hyperedge $e$ and a blue hyperedge $f$ with maximum intersection and, on contrary, suppose that $m=|e \cap f|<k-1$. Let $g$ be an edge that contains $e \cap f$ and intersects $e \backslash f$ in $\left\lfloor\frac{k-m}{2}\right\rfloor$ vertices and intersects $f \backslash e$ in $\left\lceil\frac{k-m}{2}\right\rceil$ vertices. Now, $g, e$ or $g, f$ contradicts the choice of $e$ and $f$.

Corollary 4. $R\left(\mathcal{P}_{2}^{k}, \mathcal{P}_{2}^{k}\right)=2 k-1$.

Proof. Let $\mathcal{K}_{2 k-1}^{k}$ be 2-edge colored red and blue. We may assume that $\mathcal{F}_{\text {red }}$ and $\mathcal{F}_{\text {blue }}$ are both nonempty. By Remark 3, assume that $e=\left\{v_{1}, v_{2}, \ldots, v_{k}\right\} \in \mathcal{F}_{\text {red }}$ and $e^{\prime}=\left\{v_{2}, v_{3}, \ldots, v_{k+1}\right\} \in \mathcal{F}_{\text {blue }}$. Let $W$ be the set of the remaining vertices of $\mathcal{K}_{2 k-1}^{k}$. If the $k$-set $e^{\prime \prime}=\left\{v_{1}, v_{k+1}\right\} \cup W$ is red then $e, e^{\prime \prime}$ is a red copy of $\mathcal{P}_{2}^{k}$, otherwise $e^{\prime}, e^{\prime \prime}$ is a blue copy of $\mathcal{P}_{2}^{k}$.

Theorem 5. For every $k \geqslant 3, R\left(\mathcal{C}_{3}^{k}, \mathcal{C}_{3}^{k}\right)=3 k-2$.

Proof. Suppose indirectly that the edges of $\mathcal{K}_{3 k-2}^{k}$ can be colored red and blue without monochromatic $\mathcal{C}_{3}^{k}$. Then $\mathcal{F}_{\text {red }}$ and $\mathcal{F}_{\text {blue }}$ are both nonempty, using Remark 3, select edges $e=\left\{v_{1}, v_{2}, \ldots v_{k}\right\} \in \mathcal{F}_{\text {red }}$ and $e^{\prime}=\left\{v_{2}, v_{3}, \ldots v_{k+1}\right\} \in \mathcal{F}_{\text {blue }}$. Let $v \in V\left(\mathcal{K}_{3 k-2}^{k}\right) \backslash\left(e \cup e^{\prime}\right)$ be an arbitrary vertex and let $W$ be the set of the remaining vertices. Note that $|W|=2 k-4$. Partition the vertices of $W$ into two sets $A$ and $B$ with $|A|=|B|=k-2$. The case $k=3$ can be easily settled as follows. Since there is no monochromatic $\mathcal{C}_{3}^{k}$, w.l.o.g. $e_{1}=$ $\left\{v, v_{2}, w_{1}\right\}$ is red, $e_{2}=\left\{v, v_{3}, w_{2}\right\}$ is blue, where $W=\left\{w_{1}, w_{2}\right\}$. Now, if $f=\left\{v, v_{1}, v_{4}\right\}$ is red then $e, f, e_{1}$ is red $\mathcal{C}_{3}^{k}$, otherwise, $e^{\prime}, f, e_{2}$ is blue $\mathcal{C}_{3}^{k}$. So we may assume that $k \geqslant 4$ and we prove the following claim. 
Claim. Let $T \subseteq A, S \subseteq B$ such that $|T|=t \geqslant 1,|S|=s$ and $s+t=k-2$. Let $t_{1} \in T$ and $s_{1} \in B \backslash S$ be arbitrary vertices. If $e_{1}=\left\{v, v_{2}\right\} \cup T \cup S$ is red, then $\left\{v, v_{i}\right\} \cup\left(T \backslash t_{1}\right) \cup\left(S \cup\left\{s_{1}\right\}\right)$ is red for any $v_{i} \in\left\{v_{3}, \ldots, v_{k}\right\}$.

Proof. Let $T^{\prime}=A \backslash T, S^{\prime}=B \backslash S$. Note that $\left|T^{\prime}\right|=s$ and $\left|S^{\prime}\right|=t$. We may assume that the hyperedge $e_{2}=\left\{v_{1}, v_{k+1}, v\right\} \cup T^{\prime} \cup\left(S^{\prime} \backslash s_{1}\right)$ is blue, otherwise $e, e_{1}, e_{2}$ would form a red $\mathcal{C}_{3}^{k}$. Now, If the hyperedge $e_{3}=\left\{v, v_{i}\right\} \cup\left(T \backslash t_{1}\right) \cup\left(S \cup\left\{s_{1}\right\}\right), v_{i} \in\left\{v_{3}, \ldots, v_{k}\right\}$, is red as claimed, otherwise $e^{\prime}, e_{2}, e_{3}$ would form a blue $\mathcal{C}_{3}^{k}$.

Now we return to the proof of the theorem. Without loss of generality, we may assume that the edge $e_{1}=\left\{v, v_{2}\right\} \cup A$ is red. Using the claim successively $k-2$ times switching $v_{3}$ and $v_{4}$, we obtain that $e_{1}$ and $\left\{v, v_{3}\right\} \cup B$ are both red, giving a red $\mathcal{C}_{3}^{k}$ with $e$ and the proof is finished.

Theorem 5 gives the following corollary.

Corollary 6. For every $k \geqslant 3, R\left(\mathcal{P}_{3}^{k}, \mathcal{P}_{3}^{k}\right)=R\left(\mathcal{C}_{3}^{k}, \mathcal{P}_{3}^{k}\right)=3 k-1$ and $R\left(\mathcal{S}_{3}^{k}, \mathcal{S}_{3}^{k}\right)=3 k-2$.

Proof. To prove $R\left(\mathcal{P}_{3}^{k}, \mathcal{P}_{3}^{k}\right) \leqslant 3 k-1$, suppose that the edges of $\mathcal{K}_{3 k-1}^{k}$ are arbitrary colored red and blue. By Theorem 5, we must have a monochromatic copy of $\mathcal{C}_{3}^{k}$. Without loss of generality, we may assume that $\mathcal{C}_{3}^{k} \subseteq \mathcal{F}_{\text {red }}$. Using Lemma 2 , we obtain that $\mathcal{P}_{3}^{k} \subseteq \mathcal{F}_{\text {red }}$ or $\mathcal{P}_{3}^{k} \subseteq \mathcal{F}_{\text {blue }}$, which shows that $R\left(\mathcal{P}_{3}^{k}, \mathcal{P}_{3}^{k}\right) \leqslant 3 k-1$. By the same argument, one can easily obtain that $R\left(\mathcal{P}_{3}^{k}, \mathcal{C}_{3}^{k}\right) \leqslant 3 k-1$. To see $R\left(\mathcal{S}_{3}^{k}, \mathcal{S}_{3}^{k}\right) \leqslant 3 k-2$, let the edges of $\mathcal{K}_{3 k-2}^{k}$ be arbitrary colored red and blue. By Theorem 5, we have a monochromatic, say red, copy of $\mathcal{C}_{3}^{k}$. Assume that $e_{1}=\left\{v, v_{1}, \ldots, v_{k-2}, u\right\}, e_{2}=\left\{u, u_{1}, \ldots, u_{k-2}, w\right\}$ and $e_{3}=$ $\left\{w, w_{1}, \ldots, w_{k-2}, v\right\}$ are the hyperedges of the copy of $\mathcal{C}_{3}^{k}$ and $T=\{t\}$ be the remaining vertex of $\mathcal{K}_{3 k-2}^{k}$. If one of the hyperedges $e_{1}^{\prime}=\left\{t, w, v_{1}, \ldots, v_{k-2}\right\}, e_{2}^{\prime}=\left\{t, u, w_{1}, \ldots, w_{k-2}\right\}$ or $e_{3}^{\prime}=\left\{t, v, u_{1}, \ldots, u_{k-2}\right\}$ is red, then we have a red copy of $\mathcal{S}_{3}^{k} \subseteq \mathcal{F}_{\text {red }}$, otherwise $e_{1}^{\prime} e_{2}^{\prime} e_{3}^{\prime}$ form a $\mathcal{S}_{3}^{k} \subseteq \mathcal{F}_{\text {blue }}$, which completes the proof.

To determine $R\left(\mathcal{C}_{4}^{k}, \mathcal{C}_{4}^{k}\right)$ for $k \geqslant 3$, we need the following lemma.

Lemma 7. If $\mathcal{K}_{4 k-3}^{k}$ is 2-edge colored red and blue without monochromatic $\mathcal{C}_{4}^{k}$, then there exist two pairs of red-blue hyperedges e, $e^{\prime}$ and $f, f^{\prime}$ such that $\left(e \cup e^{\prime}\right) \cap\left(f \cup f^{\prime}\right)=\emptyset$.

Proof. Set $V=V\left(\mathcal{K}_{4 k-3}^{k}\right)$. Since there is no monochromatic $\mathcal{C}_{4}^{k}, \mathcal{F}_{\text {red }}$ and $\mathcal{F}_{\text {blue }}$ are both nonempty. By Remark 3, select $e=\left\{v_{1}, v_{2}, \ldots, v_{k}\right\} \in \mathcal{F}_{\text {red }}$ and $e^{\prime}=\left\{v_{2}, v_{3}, \ldots, v_{k+1}\right\} \in$ $\mathcal{F}_{\text {blue }}$ and let $W$ denote the set of vertices uncovered by $e \cup e^{\prime}$. If $W$ is not monochromatic, we can select red-blue hyperedges $f, f^{\prime}$ in $W$ which clearly $\left(e \cup e^{\prime}\right) \cap\left(f \cup f^{\prime}\right)=\emptyset$. Thus let $W$ span a monochromatic hypergraph, w.l.o.g blue. Let $W_{1}, W_{2}$ be disjoint subsets of $W$ with cardinality $k-1$ and $w \in W \backslash\left(W_{1} \cup W_{2}\right)$ and $h_{i}=W_{i} \cup\{w\}(i=1,2)$. We may assume that $g_{1}=\left\{v_{k+1}\right\} \cup W_{1}$ is blue. Indeed, otherwise consider $g_{2}=\left\{v_{1}\right\} \cup W_{2}$. If $g_{2}$ is blue, then the red-blue pairs $e, g_{2}$ and $g_{1}, h_{1}$ satisfy the theorem. Otherwise, the red-blue pairs $g_{1}, e^{\prime}$ and $g_{2}, h_{2}$ satisfy the theorem. By symmetry, We may assume that $\left\{v_{k+1}\right\} \cup W_{2}$ is also blue. 
Next we observe that the hyperedge $f_{1}=\left\{v_{2}\right\} \cup W_{2}$, is red. If not, consider $w_{1} \in$ $W_{1}, w_{2} \in W_{2}$ and set $f^{\prime}=\left\{w_{1}, w_{2}\right\} \cup\left(W \backslash\left(W_{1} \cup W_{2}\right)\right)$. But then $e^{\prime}, f_{1}, f^{\prime}, g_{1}$ is a blue copy of $\mathcal{C}_{4}^{k}$. By a similar argument, $f_{2}=\left\{v_{3}\right\} \cup W_{1}$ must be red. However, we may assume that $b=\left\{v_{1}\right\} \cup W_{2}$ is also red, otherwise $f_{1}, b$ and $f_{2}, h_{1}$ are two disjoint red-blue pairs. Now the red-blue pairs $b, h_{2}$ and $f_{2}, e^{\prime}$ are disjoint and satisfying the theorem. This observation completes the proof.

Corollary 8. $R\left(\mathcal{C}_{4}^{3}, \mathcal{C}_{4}^{3}\right)=9$.

Proof. Suppose on the contrary that $\mathcal{K}_{9}^{3}$ is 2-edge colored red and blue without a monochromatic $\mathcal{C}_{4}^{3}$. By Lemma 7, we have two pairs of red-blue hyperedges $e, e^{\prime}$ and $f, f^{\prime}$ such that $\left(e \cup e^{\prime}\right) \cap\left(f \cup f^{\prime}\right)=\emptyset$. Using Remark 3 for $e \cup e^{\prime}$ and $f \cup f^{\prime}$, we may assume that $\left|e \cap e^{\prime}\right|=\left|f \cap f^{\prime}\right|=2$. Let $e=\left\{v_{1}, v_{2}, v_{3}\right\}, e^{\prime}=\left\{v_{2}, v_{3}, v_{4}\right\}$ and $f=\left\{w_{1}, w_{2}, w_{3}\right\}$, $f^{\prime}=\left\{w_{2}, w_{3}, w_{4}\right\}$. There is a single vertex $u$ uncovered by these four edges. W.l.o.g let $\left\{v_{2}, u, w_{2}\right\}$ be red, then $g=\left\{v_{1}, v_{4}, w_{3}\right\}$ and $h=\left\{w_{1}, w_{4}, v_{3}\right\}$ are both blue, otherwise we have a red $\mathcal{C}_{4}^{3}$. Now, $e^{\prime}, g, f^{\prime}, h$ form a blue $\mathcal{C}_{4}^{3}$, a contradiction.

Theorem 9. For every $k \geqslant 3, R\left(\mathcal{C}_{4}^{k}, \mathcal{C}_{4}^{k}\right)=4 k-3$.

Proof. The case $k=3$ follows from Corollary 8 , so we may assume that $k \geqslant 4$. Suppose indirectly that the edges of $\mathcal{K}_{4 k-3}^{k}$ can be colored red and blue without monochromatic $\mathcal{C}_{4}^{k}$. Clearly, $\mathcal{F}_{\text {red }}$ and $\mathcal{F}_{\text {blue }}$ are both nonempty. By Remark 3 and Lemma 7 , we can select $f=\left\{w_{1}, w_{2}, \ldots, w_{k}\right\} \in \mathcal{F}_{\text {red }}, f^{\prime}=\left\{w_{2}, w_{3}, \ldots, w_{k+1}\right\} \in \mathcal{F}_{\text {blue }}$ and $e=\left\{v_{1}, v_{2}, \ldots, v_{k}\right\} \in$ $\mathcal{F}_{\text {red }}, e^{\prime}=\left\{v_{2}, v_{3}, \ldots, v_{k+1}\right\} \in \mathcal{F}_{\text {blue }}$ so that $\left(f \cup f^{\prime}\right) \cap\left(e \cup e^{\prime}\right)=\emptyset$. Set $V=V\left(\mathcal{K}_{4 k-3}^{k}\right)$ and let $W=V \backslash\left(e \cup e^{\prime} \cup f \cup f^{\prime}\right)$, note that $|W|=2 k-5$. Partition the vertices of $W$ into two sets $A$ and $B$ with $|A|=|B|=k-3$ and let $u$ be the remaining vertex of $W$. In the following claims, we let $T \subseteq A, S \subseteq B$ such that $|T|=t \geqslant 1,|S|=s$ and $s+t=k-3$. Also let $t_{1} \in T$ and $s_{1} \in B \backslash S$ be arbitrary. Set $T^{\prime}=A \backslash T, S^{\prime}=B \backslash S$, note that $\left|T^{\prime}\right|=s$ and $\left|S^{\prime}\right|=t$.

Claim 1. If $i \in\{2,3, \ldots, k\}$ and $e_{1}=\left\{w_{1}, w_{k+1}, v_{i}\right\} \cup T \cup S$ is red, then for any $j \in\{2,3, \ldots, k\}$, the hyperedge $g_{1}=\left\{v_{1}, v_{k+1}, w_{j}\right\} \cup\left(T \backslash t_{1}\right) \cup\left(S \cup\left\{s_{1}\right\}\right)$ is also red.

Proof. Let $m \in\{2,3, \ldots, k\} \backslash\{i\}$. The hyperedge $f_{1}=\left\{u, v_{m}, w_{j}\right\} \cup T^{\prime} \cup S^{\prime}$ is blue, otherwise $e, f_{1}, f, e_{1}$ form a red $\mathcal{C}_{4}^{k}$. By a similar argument, for $i \neq j, h=\left\{v_{1}, v_{k+1}, w_{i}\right\} \cup$ $T \cup S$ is red (otherwise $e^{\prime}, f^{\prime}, f_{1}, h$ is blue $\left.\mathcal{C}_{4}^{k}\right)$ and $f_{2}=\left\{u, w_{1}, w_{k+1}, v_{i}\right\} \cup T^{\prime} \cup\left(S^{\prime} \backslash s_{1}\right)$ is blue (otherwise $e, f, h, f_{2}$ is red $\mathcal{C}_{4}^{k}$ ). Now, if $g_{1}$ is blue then $e^{\prime}, g_{1}, f^{\prime}, f_{2}$ is a blue $\mathcal{C}_{4}^{k}$, a contradiction, thus $g_{1}$ is red as claimed.

By symmetry, we have the following claim, which is given without proof.

Claim 2. If $i \in\{2,3, \ldots, k\}$ and $e_{1}=\left\{v_{1}, v_{k+1}, w_{i}\right\} \cup T \cup S$ is red, then for any $j \in\{2,3, \ldots, k\}$, the hyperedge $g_{1}=\left\{w_{1}, w_{k+1}, v_{j}\right\} \cup\left(T \backslash t_{1}\right) \cup\left(S \cup\left\{s_{1}\right\}\right)$ is also red.

To finish the proof, we need the following. 
Claim 3. If $e_{1}=\left\{v_{2}, w_{2}, u\right\} \cup T \cup S$ is red, then $g_{1}=\left\{w_{1}, w_{k+1}, v_{i}\right\} \cup\left(T \backslash t_{1}\right) \cup\left(S \cup\left\{s_{1}\right\}\right)$ is red for any $i \in\{3,4, \ldots, k\}$.

Proof. Let $i \in\{3,4 \ldots, k\}$. The hyperedge $f_{1}=\left\{v_{1}, v_{k+1}, w_{j}\right\} \cup T^{\prime} \cup S^{\prime}$ is blue for for any $j \in\{3,4, \ldots, k\}$, otherwise $e, f_{1}, f, e_{1}$ would form a red $\mathcal{C}_{4}^{k}$. Then $h=\left\{w_{1}, w_{k+1}, v_{i}\right\} \cup T \cup S$ is red otherwise $e^{\prime}, f_{1}, f^{\prime}, h$ would give a blue $\mathcal{C}_{4}^{k}$. The edge $f_{2}=\left\{v_{1}, v_{k+1}, w_{j}, u\right\} \cup T^{\prime} \cup$ $\left(S^{\prime} \backslash s_{1}\right)$ is blue otherwise $e^{\prime}, f_{2}, f^{\prime}, h$ is a blue $\mathcal{C}_{4}^{k}$. Now, if $g_{1}$ is blue then $e^{\prime}, g_{1}, f^{\prime}, f_{2}$ is a blue $\mathcal{C}_{4}^{k}$, a contradiction. Therefore $g_{1}$ is red as claimed.

Now we finish the proof of the theorem. Without loss of generality, let $e_{1}=\left\{v_{2}, w_{2}, u\right\} \cup$ $A$ be red. Using Claim 3, $\left\{w_{1}, w_{k+1}, v_{i}\right\} \cup\left(T \backslash t_{1}\right) \cup\left(S \cup\left\{s_{1}\right\}\right), i \in\{3,4, \ldots, k\}$, is red and so using Claim 1 and Claim 2 successively $k-3$ times and switching the pairs $\left(v_{3}, w_{3}\right),\left(v_{4}, w_{4}\right)$ (starting with $t=k-3$ ), we obtain that the edge $h_{1}=\left\{v_{1}, v_{k+1}, w_{3}\right\} \cup B$ is red, a contradiction, since $e, e_{1}, f, h_{1}$ is a red $\mathcal{C}_{4}^{k}$. This observation completes the proof.

From Lemma 2 and Theorem 9 we get the following.

Corollary 10. For every $k \geqslant 3, R\left(\mathcal{P}_{4}^{k}, \mathcal{P}_{4}^{k}\right)=R\left(\mathcal{C}_{4}^{k}, \mathcal{P}_{4}^{k}\right)=4 k-2$.

Proving $R\left(\mathcal{C}_{3}^{k}, \mathcal{C}_{3}^{k}, \mathcal{C}_{3}^{k}\right)=3 k-1$ for $k \geqslant 3$ seems difficult, we could do it only for $k=3$.

Theorem 11. $R\left(\mathcal{C}_{3}^{3}, \mathcal{C}_{3}^{3}, \mathcal{C}_{3}^{3}\right)=8$.

Proof. We suppose that the third color is green and the vertex set is $V$. The lower bound is obtained by coloring triples of $\{1,2, \ldots 7\}$ by red if the smallest element in the triple is 1 , blue if the smallest element in the triple is 2 and green otherwise. To see the upper bound, we consider some cases.

Case 1. There is a monochromatic, say green $S=\mathcal{S}_{3}^{3}$ with edges $e_{1}=\{A, 1,4\}, e_{2}=$ $\{A, 2,5\}$ and $e_{3}=\{A, 3,6\}$.

There is one vertex, $B$, not covered by $S$. Set $U=\{1,2,3\}, W=\{4,5,6\}$ and let $H$ be the graph obtained from the complete graph spanned by $U \cup W$ by removing the pairs $14,25,36$. Clearly, for any edge $i j$ of $H$, the hyperedge $\{B, i, j\}$ is red or blue, otherwise we have a green $\mathcal{C}_{3}^{3}$. For any edge $i j$ of $H$ we define a mirror edge $k l$ so that $k$ is the third vertex in the edge of $S$ containing $\{A, i\}$ and $l$ is the third vertex of the edge of $S$ containing $\{A, j\}$.

Subcase 1.1. For any edge $i j$ of $H$ and its mirror edge $k l$, the hyperedges $\{i, j, B\},\{k, l, B\}$ have different colors (one of them red and the other is blue).

Among the hyperedges $\{1,5, B\},\{2,6, B\},\{3,4, B\}$ two colored with the same color, say the first two is colored with red. By the subcase assumption, the hyperedges 
$\{2,4, B\},\{3,5, B\}$ are blue. Now the hyperedge $f=\{4,5,6\}$ defines a monochromatic $\mathcal{C}_{3}^{3}$ : if green, with $e_{1}, e_{2}$; if red, with $\{1,5, B\},\{2,6, B\}$; if blue, with $\{2,4, B\},\{3,5, B\}$.

Subcase 1.2. There exists an edge of $H$, say 15 , such that with its mirror edge 24 , the hyperedges $\{1,5, B\},\{2,4, B\}$ are both red.

We claim that for any vertex $v \in V \backslash\{1,2,4,5\}$ (there are four such vertices) the hyperedges $\{1,5, v\},\{2,4, v\}$ are red, the hyperedges $\{1,4, v\},\{2,5, v\}$ are green and the hyperedges $\{1,2, v\},\{4,5, v\}$ are blue. We call such a quadruple $Q=\{1,2,4,5\}$ a factored quadruple. First observe that the hyperedges $\{1,2,3\},\{1,2,6\},\{4,5,3\}$ and $\{4,5,6\}$ are blue, otherwise we have a monochromatic $\mathcal{C}_{3}^{3}$. Similarly, the hyperedges $\{1,4,6\},\{2,5,6\}$ are green. These observations with the initial assumptions are easily imply that $Q$ is factored from $A$ as required. An easy consequence is that $Q$ is factored from the other three vertices in the same way.

At this point we introduce new notations, consider a factored quadruple $Q=$ $\left\{A_{1}, A_{2}, A_{3}, A_{4}\right\}$ and its complement $R=V \backslash Q=\{1,2,3,4\}$. Two of the four hyperedges in $R$ have the same color, say $\{1,2,3\},\{2,3,4\}$ are green. Let $G_{R}$ be the graph with vertex set $R$ and with all edges except 23. Observe that for any edge $i j \in E\left(G_{R}\right)$, the hyperedge $f=\left\{A_{k}, i, j\right\}$ is red or blue. Indeed, otherwise consider the green hyperedge $e$ in $R$ that splits $i j$, say $i \in e, j \notin e$. Since $Q$ is factored, there is a green hyperedge $g$ with one vertex in $e \backslash\{i\}$ that intersects $Q$ in two vertices and $A_{k} \in g$. The edges $e, f, g$ would form a green $\mathcal{C}_{3}^{3}$.

Now we may assume that $e=\left\{1,4, A_{1}\right\}$ is red. Since $Q$ is factored, there is $A_{i} \neq$ $A_{1}$, say $A_{2}$, such that $\left\{j, A_{1}, A_{2}\right\}$ is red for all $j \in R$. If $f=\left\{1,3, A_{2}\right\}$ is red, then $e, f,\left\{2, A_{1}, A_{2}\right\}$ would form a red $\mathcal{C}_{3}^{3}$. Thus $f$, and similarly $g=\left\{2,4, A_{2}\right\}$ is blue. Since $Q$ is factored, with one of $A_{3}, A_{4}$, say with $A_{3},\left\{j, A_{2}, A_{3}\right\}$ is blue for all $j \in R$. Now $\left\{1,2, A_{3}\right\}$ must be red, otherwise $\left\{1,2, A_{3}\right\}, f=\left\{1,3, A_{2}\right\},\left\{4, A_{2}, A_{3}\right\}$ would form a blue $\mathcal{C}_{3}^{3}$. Similarly $\left\{3,4, A_{3}\right\}$ must be red and so hyperedges $\left\{1,2, A_{3}\right\},\left\{3,4, A_{3}\right\},\left\{1,4, A_{1}\right\}$ form a red $\mathcal{C}_{3}^{3}$.

Case 2. There is no monochromatic $S=\mathcal{S}_{3}^{3}$.

It is easy to see (also follows from a well-known result [1]) that (centered at any vertex A) there is a monochromatic, say green, $T=\mathcal{S}_{2}^{3}$, with edges $\{A, 1,2\},\{A, 3,4\}$. Let $U=\{5,6,7\}$ denote the set of vertices uncovered by $T$.

Subcase 2.1. There is a monochromatic (red or blue) $\mathcal{S}_{2}^{3}$ in the form $\{i, 1,3\},\{i, 2,4\}$ or $\{i, 1,4\},\{i, 2,3\}, i \in U$.

Suppose w.l.o.g that the hyperedges $\{i, 1,3\},\{i, 2,4\}$ are red. Then $\{j, 1,4\},\{j, 2,3\}$ must be blue for $j \in U, j \neq i$, otherwise we have green or red $\mathcal{C}_{3}^{3}$. Then no matter what is the color of the edge $\{A, i, j\}$, we have a monochromatic $\mathcal{S}_{3}^{3}$ and this leads back to the Case 1. 
Subcase 2.2. For every $i \in U$ the pairs of edges $\{i, 1,3\},\{i, 2,4\}$ and $\{i, 1,4\},\{i, 2,3\}$ have different (red-blue) colors.

From the subcase definition w.l.o.g. we find two edges $\{1,3, i\},\{2,3, j\}$ of the same color, say red, such that $i, j \in U, i \neq j$. This implies that $\{A, i, j\}$ is blue (green would give green $\mathcal{S}_{3}^{3}$, red would give red $\mathcal{C}_{3}^{3}$ ). The edge $\{2,4, i\}$ is also blue (green or red both would give a monochromatic $\mathcal{C}_{3}^{3}$ ). No matter what is the color of $\{1,4, j\}$, a monochromatic $\mathcal{C}_{3}^{3}$ arises.

\section{Concluding remarks}

It would be interesting to decide whether the natural lower bound of Lemma 1 is always the true value of the Ramsey numbers for $k$-uniform (loose) paths and cycles if $k \geqslant 3$. The test case is $k=3$.

Question 12. For every $n \geqslant m \geqslant 2, R\left(\mathcal{P}_{n}^{3}, \mathcal{P}_{m}^{3}\right)=R\left(\mathcal{P}_{n}^{3}, \mathcal{C}_{m}^{3}\right)=R\left(\mathcal{C}_{n}^{3}, \mathcal{C}_{m}^{3}\right)+1=$ $2 n+\left\lfloor\frac{m+1}{2}\right\rfloor$ ? In particular,

$$
R\left(\mathcal{P}_{n}^{3}, \mathcal{P}_{n}^{3}\right)=R\left(\mathcal{C}_{n}^{3}, \mathcal{C}_{n}^{3}\right)+1=\left\lceil\frac{5 n}{2}\right\rceil ?
$$

We answer this question affirmatively when one of $m, n$ is small, at most 6 , in a paper under preparation [7].

In certain cases one could get estimates of the Ramsey numbers addressed in this paper by applying Turán-type results. For example, the behavior of the $r$-color Ramsey number of triangles in hypergraphs is essentially different from the graph case. While $R\left(C_{3}, C_{3}, \ldots, C_{3}\right)$ is at least exponential in $r$ (for $r$ arguments), it is linear for hypergraphs.

Proposition 13. For $r$ arguments, $r+5 \leqslant R\left(\mathcal{C}_{3}^{3}, \mathcal{C}_{3}^{3}, \ldots, \mathcal{C}_{3}^{3}\right) \leqslant 3 r+1$.

Proof. The lower bound is obtained by coloring triples of $\{1,2, \ldots r+4\}$ by their smallest element $i$ if $i<r$, otherwise by color $r$. The upper bound follows from the Turán number of $\mathcal{C}_{3}^{3}$ (see [2]): If $n \geqslant 6, \mathcal{F} \subseteq\left(\begin{array}{l}n \\ 3\end{array}\right)$ and $\mathcal{F}$ contains no $\mathcal{C}_{3}^{3}$, then $|\mathcal{F}| \leqslant\left(\begin{array}{c}n-1 \\ 2\end{array}\right)$. Therefore, for the smallest $n$ for which

$$
\left(\begin{array}{l}
n \\
3
\end{array}\right)>r\left(\begin{array}{c}
n-1 \\
2
\end{array}\right)
$$

every $r$-colored $\mathcal{K}_{n}^{3}$ will contain a monochromatic $\mathcal{C}_{3}^{3}$, implying the upper bound.

It is known from [2] that if $n \geqslant 8, \mathcal{F} \subseteq\left(\begin{array}{l}n \\ 3\end{array}\right),|\mathcal{F}|=\left(\begin{array}{c}n-1 \\ 2\end{array}\right)$ and $\mathcal{F}$ contains no $\mathcal{C}_{3}^{3}$, then all elements of $\mathcal{F}$ goes through a fix point. Using this fact, the upper bound of the previous proposition can be reduced to $3 r$ for $r \geqslant 3$. We proved here that for $r=2,3$ the lower bound is the truth and we suspect that this is true for all $r$. 


\section{References}

[1] E.J. Cockayne, P.J. Lorimer, The Ramsey number for stripes, J. Austral. Math. Soc. 19 (1975), 252-256.

[2] R. Csákány, J. Kahn, A homological approach to two problems on finite sets, J. Algebraic Combin. 9 (1999), 141-149.

[3] R.J. Faudree, S.L. Lawrence, T.D. Parsons, R.H. Schelp, Path-Cycle Ramsey Numbers, Discrete Math. 10 (1974), 269-277.

[4] R.J. Faudree and R.H. Schelp, All Ramsey Numbers for Cycles in Graphs, Discrete Math. 8 (1974), 313-329.

[5] L. Gerencsér, A. Gyárfás, On Ramsey-Type Problems, Annales Universitatis Scientiarum Budapestinensis, Eötvös Sect. Math. 10 (1967), 167-170.

[6] A. Gyárfás, G. Sárközy, E. Szemerédi, The Ramsey number of diamond-matchings and loose cycles in hypergraphs, Electron. J. Combin. 15 (2008), no. 1, \#R126.

[7] A. Gyárfás, G. Raeisi, Some Ramsey numbers for loose cycles and paths in 3-uniform hypergraphs, in preparation.

[8] P. Haxell, T. Luczak, Y. Peng, V. Rödl, A. Ruciński, M. Simonovits, J. Skokan, The Ramsey number for hypergraph cycles I, J. Combin. Theory, Ser. A, 113 (2006), $67-83$. 